\title{
Improving on Phase Contrast by Extending the Range of Captured Spatial Frequencies in Transmission Electron Microscopy
}

\author{
C. Kisielowski
}

National Center for Electron Microscopy and Helios SERC, Lawrence Berkeley National Laboratory, One Cyclotron Rd., Berkeley CA 94720, USA

In recent years materials research rapidly expanded to include bioinspired composites, new thin films and membranes such as carbon-based materials of exceptional strength, or soft ordered organic films of molecular thickness and exceptional flexibility. Bioinspired polymers and functionalized nanocrystals forming core-shell structures fill the gap between these extremes. As a result, materials research nowadays strives to take advantage of these rich developments by aiming at designed functionality at the molecular level. The Helios effort at LBNL to convert sunlight to fuel may serve as an example since it represents a quest to design an artificial system of medium complexity. Clearly, a further development of electron microscopy is desirable to help investigating this rich variety of new materials and one aspect of this quest is the enhancement of contrast.

Electron microscopy has now pushed resolution to deep sub-Ångstrom values [1] by accessing higher spatial frequencies. In fact, resolution has now reached limits that are given at a fundamental level by the Coulomb scattering process, and instrument performance is often not longer the limiting factor [2]. The ability to record high spatial frequencies to beyond $0.05 \mathrm{~nm}$ yields a significant increase of detection limits and signal-to-noise ratios, which made it possible to detect single atoms across the Periodic Table of Elements even if light atoms are involved [3]. Acceleration voltages can be chosen between $300 \mathrm{kV}$ and $50 \mathrm{kV}$ and possibly below to minimize beam-sample interactions while maintaining atomic resolution. Similarly, progress was made to capture low spatial frequencies using phase plates [4]. The Figures $1 \mathrm{a}$ and $1 \mathrm{~b}$ show that a signal enhancement by a factor of $\sim 5$ was already achieved utilizing a Boersch phase plate that captures spatial frequencies between $1 / 10 \mathrm{~nm}^{-1}$ and $1 / 0.2 \mathrm{~nm}^{-1}$ at $200 \mathrm{kV}$. Further, the contrast in Fig. $1 \mathrm{~b}$ is comparable to what is currently possible by capturing high spatial frequencies between $1 \mathrm{~nm}^{-1}$ and $1 / 0.1 \mathrm{~nm}^{-1}$ or above with the TEAM 0.5 microscope [5] operated at $80 \mathrm{kV}$ (Fig. 1c). However, the significantly larger resolution in Figure 1c is obtained by compromising the low spatial frequency transfer. It is remarkable that the contrasts in the images of Figures $1 \mathrm{~b}$ and 1c are well comparable even though the sampled spatial frequencies differ. Clearly, a contrast optimized information transfer should allow for capturing the entire spatial frequency spectrum. One approach to achieve this goal consists of making use of unique properties of Cc correction [6], which is available in the TEAM I microscope [1]. It is seen from Figure 2 that it has indeed become possible to capture the spatial frequency range from $1 / 9 \mathrm{~nm}^{-1}$ to $1 / 0.1 \mathrm{~nm}^{-1}$ into a single image. Advantages and drawbacks of different approaches will be discussed. [7]

1. U. Dahmen, R. Erni, V. Radmilovic, C. Kisielowski, et al., $\square$ Phil. Trans. R. Soc. A 367, (2009) 3795-3808

2. C. Kisielowski, P. Specht, R. Erni, Q.M. Ramasse, in Frontiers of Characterization and Metrology for Nanoelectronics,

D.G. Seiler, al., (eds.), American Institute of Physics Conference Proceedings 1173 (2009) 231

3. Ç.Ö. Girit, J.C. Meyer, R. Erni, M. D. Rossell, C. Kisielowski, L. Yang, C.H. Park, et al. Science 323 (2009) 1705

4. D. Alloyeau, W.K. Hsieh, E.H. Anderson, D.L. Hilken, X. Meng, et. al., Ultramicroscopy (2010), in press

5. C. Kisielowski, B. Freitag, M. Bischoff, H. van Lin, S. Lazar, et al., Microscopy and Microanalysis 14, (2008) 454

6. For details see: A. Kirkland, C. Kisielowski et al., these proceedings

7. This work was supported by the Electron Microscopy of Soft Matter Program under DoE's contract no. DE-AC02-

05CH11231. Microscopy was performed at the NCEM. 

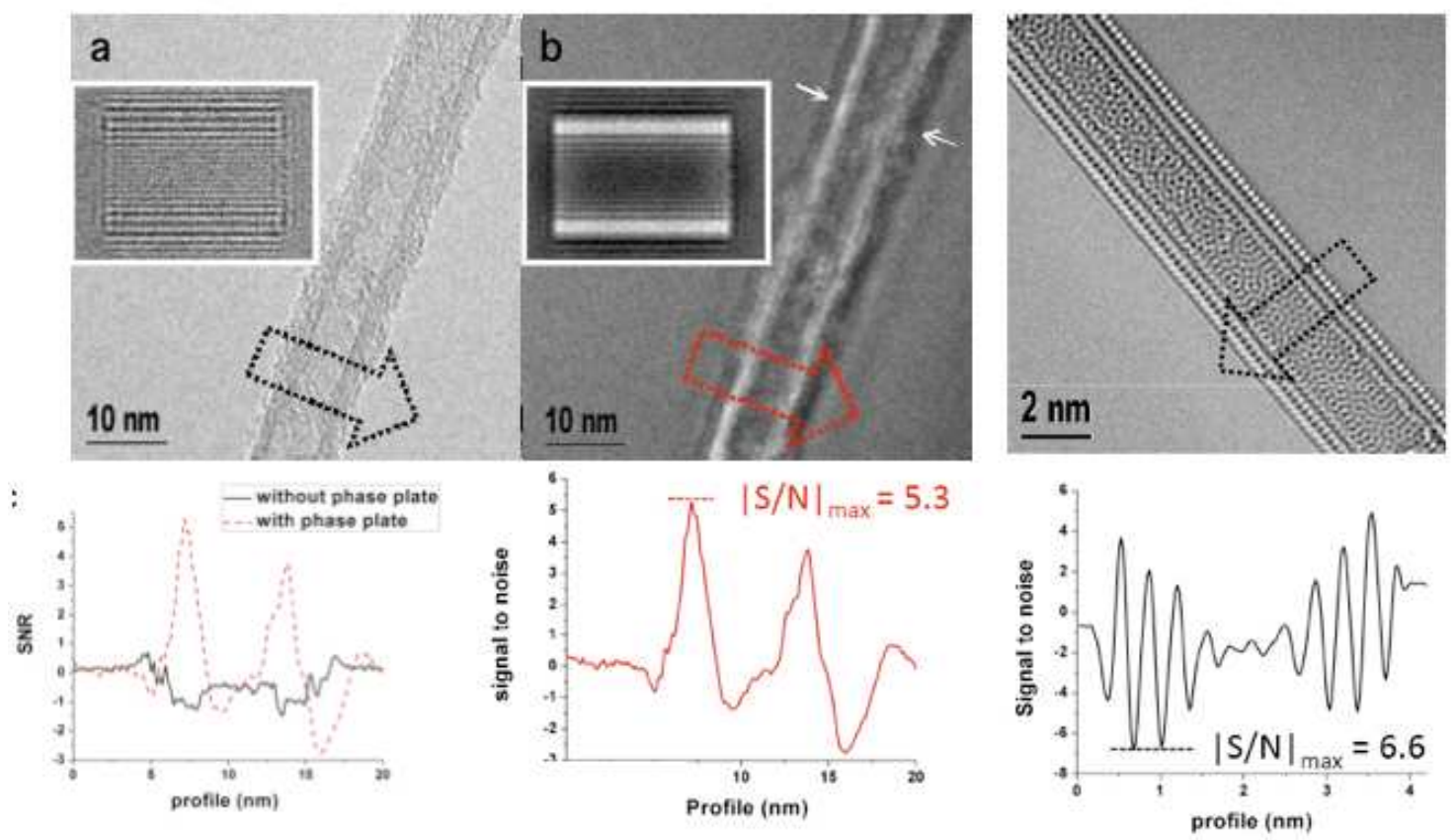

FIG. 1. Contrast enhancement in images of multi-wall carbon nanotubes. a) In focus image recorded at $200 \mathrm{kV}$ with a Libra 200 microscope. b) as a) but with a Boersch phase plate activated causing a $90^{\circ}$ phase shift. c) Object imaged with TEAM 0.5 operated at $80 \mathrm{kV}$. Bottom: Integrated line profiles as indicated in the images [4]

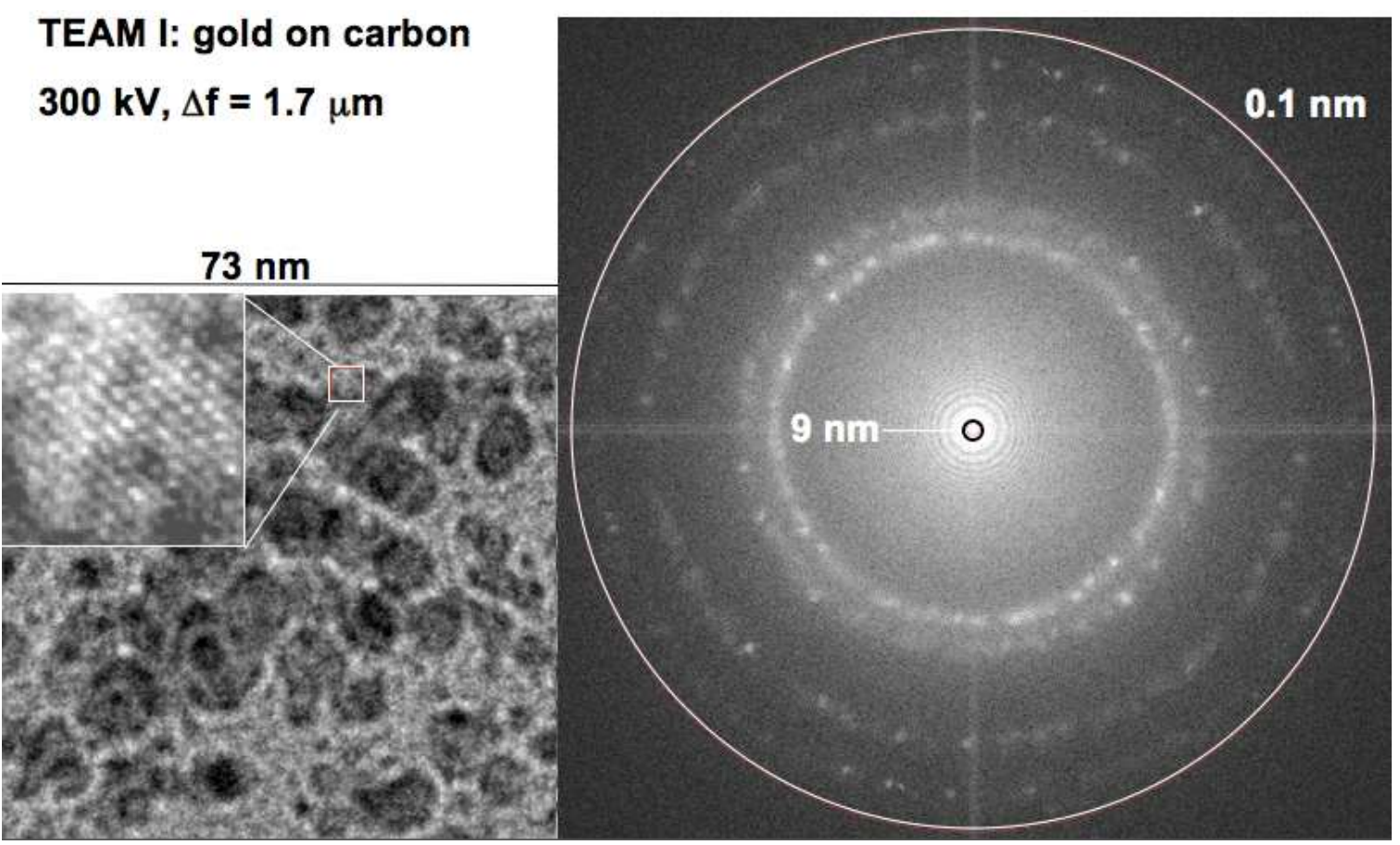

FIG. 2. Information transfer in the $\mathrm{Cc} \& \mathrm{Cs}$ corrected TEAM I differs from that of conventional microscopes even at large defocus values $\Delta \mathrm{f}$. Left: Defocused phase contrast image with the inset showing that atomic resolution is maintained. Right: Fourier transform with Thon rings in the center. Spatial frequencies between $1 / 9 \mathrm{~nm}^{-1}$ and $1 / 0.1 \mathrm{~nm}^{-1}$ are captured in a single image. 